\title{
Dinámica epidemiológica de la escarlatina en Chile
}

\author{
Dr. Mauricio Canals L.'
}

\author{
Epidemiological dynamics of scarlet fever in Chile
}

\begin{abstract}
The monthly incidence of scarlet fever was studied by time-series analysis. Two types of periodicfluctuations were detected involving a birnodal short seasonal cycle and a long-term cycle whose perio $d$ takes 5 years aproximately. The interactions of the respiratory transmition mechanism, person to person oontacts and environmental temperature would explain the seasonal cycle bimodality. The long term cycle would be explained by asinchronic fluctuations of the fraction of population assigned to "cases" and "susceptibles".
\end{abstract}

(Key words: scarlet fever, epidemiology.)

Una de las manifestaciones de la interacción entre las poblaciones humanas y las poblaciones de Streptococcus $s p$. es la escarlatina.

Es una enfermedad frecuente en Chile, cuya incidencia mensual es alrededor de 4 por 100.000 , afectando fundamentalmente a los niños ${ }^{1-3}$. Sin embargo, a pesar de su frecuencia y tal vez por ella, existen pocos estudios de su dinámica de infección.

Existen estudios teóricos ${ }^{4-7}$ basados en datos empíricos ${ }^{8-12}$ que demuestran fluctuaciones estacionales en la incidencia de enfermedades infecciosas y que sugieren una recurtencia epidémica periódica.

El objetivo de este trabajo es estudiar la incidencia de la escarlatina en Chile buscando oscila. ciones periódicas tanto estacionales como secula. res y las posibles causas de ellas.

\section{MATERIAL Y METODOS}

La fuente de información la constituyen los Anuarios de Enfermedades de Notificación Obligatoria entre los atios 1954 y 1984 .

Se analizaron las tasas de incidencia mensual de escarlatina en Chile entre estos atios.

La serie de incidencias se estudió corrigiéndole la tendencia lineal, sustrayéndole a los valores observados los valores predichos a través de la recta de regresión mínimo cuadrática.

1. Departamento de Ciencias Ecológicas, Facultad de Ciencias U. de Chile.
El método de análisis consistió en el estudio de la función de autocorrelación (correlograma) y análisis armónico de Fourier (periodograma) ${ }^{13}$. El primer método consiste en estudiar la correlación temporal de gas incidencias variando el orden de ellas, de tal manera que se obtienen correlaciones máximas cuando coincide el orden con el período de oscilación propio de la serie analizada E1 segundo método busca encontrar la o las frecuencias que explican la vartabilidad de la serie.

El significado estadístico de los resultados se estudió a través de los límites de confanza del 95\% en el periodograma acumulado.

\section{RESULTADOS}

La figura 1 muestra la incidencia mensual de escarlatina en Chile entre los años 1954 y 1984. Se observa una tendencia ascendente alcanzando en 1984 una incidencia mensual de aproximadamente 5 por 100.000 .

La figura 2 muestra la distribución mensual de la incidencia de escarlatina en los treinta antos considerados en este estudio, destacándose un patrón estacional bimodal de esta enfermedad, con incidencias máximas de mayo y noviembre.

La figura 3 muestra el correlograma de la serie una vez corregida la tendencia. Se observan ciclos con sectores de alta correlación centrados entre los 60 y 70 meses, una fluctuación con máximos relativos en los órdenes múltiplos de 12 y picos secundarios en los ordenes múltiplos de 6 . Estos hallazgos sugieren periodicidades estacionales (6 y 12 meses) y seculares (alrededor de 5 años). 


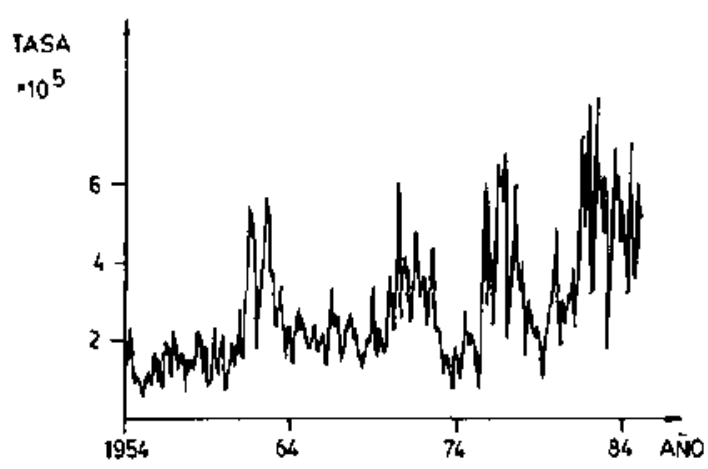

Figura 1: Tasa de íncidencia menstual de escarlatina en Chile. 1954-1984.

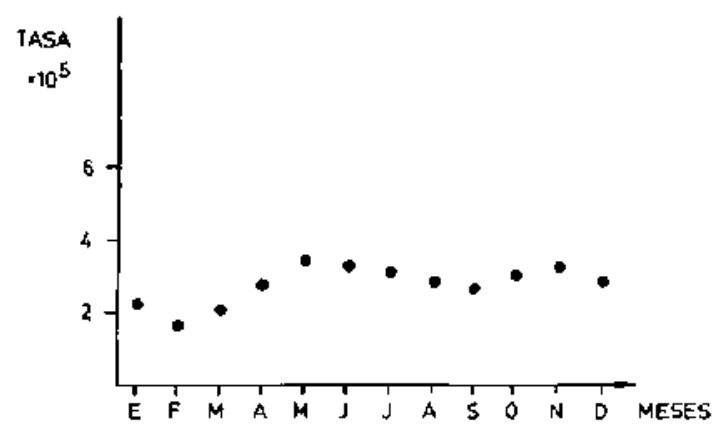

Figura 2: Distribución mensual de la incidencia media de escarlatina en Chile. 1954-1984.

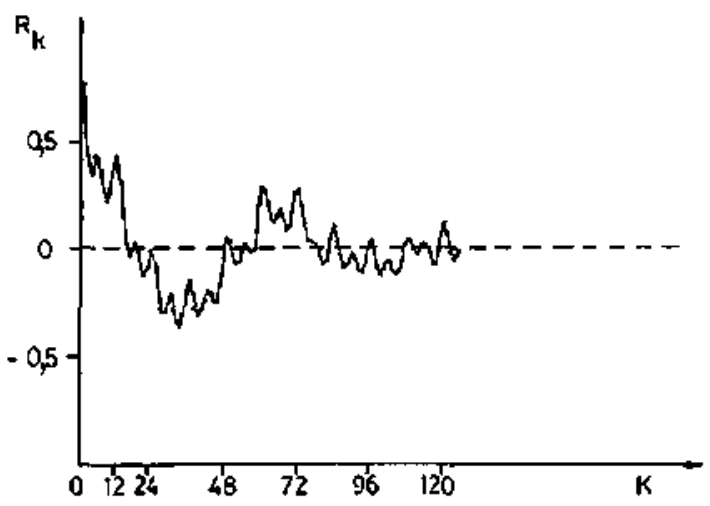

Figura 3: Correlograma te la incidencia mensual de escarlatina. (Rk: Autocorrelación; k: Orden).
La figura 4 muestra el periodograma de la misma serie. Se observan máxìmos relativos en las frecuencias 0,$0267 ; 0,083$ y 0,167 meses $^{-1}$, to que indica períodos involucrados de aproximadamente $60(=1 / 0,0167), 12$ y 6 meses, coincidiendo con lo indicado por el correlograma.

La figura 5 muestra el periodograma acumulado de la serie. Se observa que el comportamiento de la incidencia difjere de un proceso puramente aleatorio excediendo los límites de confianza del 95\% de Kolmogorov-Smirnov.

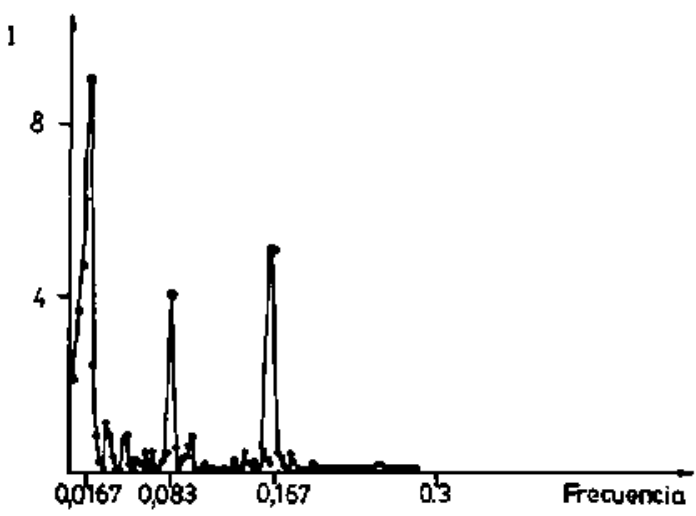

Figura 4: Periodograma de la incidencia mensual de escarlatina. (I: Intensidad en unidades relativas, Frecuencia en meses).

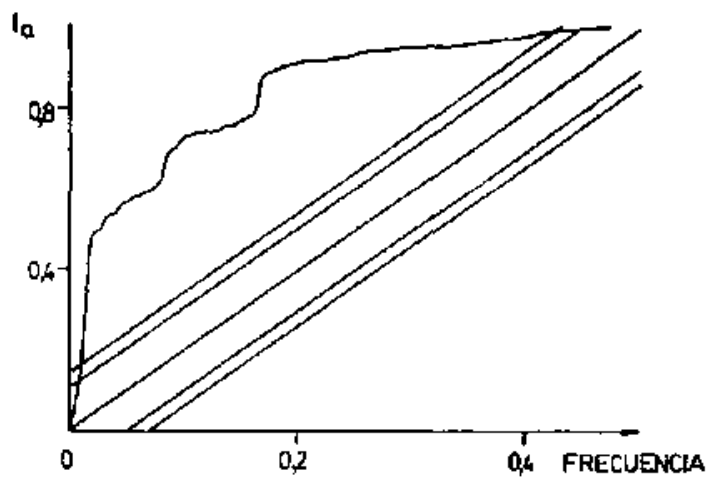

Figura 5: Periodograma acumulado de la incidencia mensual de escarlatina. (Ia: Intensidad acumulada, Frecuencia en meses).

\section{DISCUSION}

Hemos encontrado fluctuaciones estacionales $y$ seculares. 
Las fluctuaciones estacionales han sido citadas para múltiples enfermedades infecciosas ${ }^{8-10,12}$. Como causa de ellas se han invocado factores clímaticos tales como la temperatura ambiente y la humedad relativa, factores sociales tales como la agregación entre los individuos y facto. res propios del huésped tales como el estado inmunitario $^{10,11}$. Se considera que las enfermedades de transmisión respiratoria (entre ellas la escarlatina) tendrían mayor incidencia invernal; sin embargo, algunos autores han encontrado diferencias respecto a esta afirmación ${ }^{3}$.

Hemos encontrado en la escarlatina un curioso patrón estacional bimodal con incidencias máximas en mayo y noviembre (figura 2). Este patrón puede estar explicado por la confluencia de varios factores. Por un lado, la importancia del contacto persona a persona, la alta contagiosidad y el ser los niños el núcleo de mantención de la enfermedad ${ }^{2,3}$, favorecerían la alta incidencia en los meses correspondientes al período escolar, $y$ por otro, la influencia de las bajas temperaturas en la capacidad de sobrevivencia del microbio explicaría el descenso invernal.

Existen referencias que señalan la presencia de recurrencia epidémica periódica en la escarlatina habiéndose mencionado ciclos de 4,4 años $^{8}$ y de alredeơor de 6 años ${ }^{14}$. Nuestra observación cae en el rango que describen estas cifras (60 meses). Esta recurrencia epidémica se atribuye a la fluc. tuación asincrónica de las fracciones de la pobla. ción que corresponden a casos y susceptibles, hecho que ocurre de tal manera que un ascenso en el número de casos provoca agotamiento de los susceptibles, con la consiguiente caida de los casos. El período de las fluctuaciones seculares está relacionado con la edad media de infección (A) y el tiempo promedio entre la adquisición de la enfermedad y el contagio $(\tau)$, existiendo un periodo esperado ( $\mathrm{T}$ ) basado en la ecuación:

$$
\mathrm{T}=2 \cdot \pi \cdot(\mathrm{A} \cdot \tau)^{1 / 2}
$$

Se esperaría un ciclo de 4 años para la escarlatina a partir de esta ecuación ( $\mathrm{A}=7,7$ años, basados en nuestros datos y $t=19$ días $^{8}$ ). Aunque la diferencia con nuestra observación no es exagerada, ésta puede estar dada porque la ecuación citada tiene como uno de sus supuestos la mezcla homogénea de la población, hecho improbable en nuestro país.
El patrón de comportamiento de la escarlatina en Chile tiene, entonces, dos caracteristicas que lo hacen muy particular dentro de las enfemedades infecciosas. Uno es el patrón estacional bimodal no descrito para otras enfermedades infecciosas y, el otro, la recurrencia periódica. Este último hecho es esperable en enfermedades que presentan un fuerte grado de compromiso entre las poblaciones del agente y del hospedero. Este se establece, generalmente, a través de la exclusividad del hospedero, gran virulencia $y, o$, inmunidad permanente. En el caso de la escarlatina no hay hospedero único para el estreptococo ni tiene, la enfermedad, gran mortalidad, por lo que el "compromiso" agente-hospedero se establece exclusivamente a través de la inmunidad antitóxica permanente.

\section{RESUMEN}

Basados en la información contenida en los Anuarios de Enfermedades de Notificación Obligatoria se analizaron las fluctuaciones en la incidencia mensual de la escarlatina en Chile mediante análisis de series de tiempo.

Se detectan fluctuaciones periódicas de la incidencia que involucran un ciclo corto estacional bimodal y un ciclo largo, secular, de aproximadamente 5 años.

La fluctuación estacional se atribuye a la interacción agente-huésped-ambiente, destacando la importancia del mecanjsmo de transmisión aérea y del contacto persona a persona, los que en conjunto explicarían la bimodalidad. La fluctuación secular se debería a la variación asincrónica de los casos y susceptibles.

\section{AGRADECIMIENTOS}

A los miembros del laboratorio de Ecorisiología de Vertebrados, Departamento de Ecología, Facultad de Ciericias, por su colaboración.

A la doctora Lucía Cifuentes, por la revisión crítica de este trabajo.

\section{REFERENCIAS}

1. Anónimo: Anuarios de enfermedades de notificación obligatoria. Ministerio de Salud, Chile. 19541984.

2. Armijo R.: Enfermedades estreptocócicas en Armijo R. Epidemiología II. Intermédica B. Aires, Argentina. 1974; $227 \cdot 231$. 
3. Medine E., Kaempffer $A_{+}$, Kirschbaum $A_{\text {, Carras- }}$ co R., Pascual $J$. y Medino R.: Enfermedades infecciosas en Chile: Situación actual y perspectivas. Rev Méd Chile 1985; 113: 369-375.

4. Anderson R, y May R.: Population biology of infectious diseases: Part I. Nature 1979; 280; $361-367$.

5. Bartlett M.: Masles Periodicity and community size. J. R. Stat Soc A $1957 ; 120: 48-58$.

6. Bayley $N$.: Recurrent epidemics and endemicity, en Bayley $N$. The mathematical theory of infectious diseases and its applications. Charles Griftin \& Company. 1975; 133-156.

7. Soper $\boldsymbol{H}$.: Interpretation of periodicity in disease prevalence. J R. Stat So c A 1929;92: 34-73.

8. Anderson R., Grenfell B, y May R.: Oscillatory fluctuations in the incidence of infectious diseases and the impact of vaccination: time series analysis. J Hyg Camb 1984; 93:587-608.

9. Fine P. y Clarkson I.: Measles in England and
Wales $I$ : an analysis of factors underlying seasonal patterns. Int Jour Ep 1982; 11: 5-14.

10. Fine $P, y$ Clarkson $f .:$ Seasonal influences on Pertussis. Int Jour Ep 1986; 15: 237-247.

11. Yorke $J$, y London $W$.: Recurrent outbreaks of measles, chikenpox and mumps. Systematic differences in contact rates and stocastic effects. Amer J Ep 1973; 98: 469-482.

12. Yorke J.. Nathanson N., Panigiani G. y Martin J.: Seasonality and the requirements for perpetuation and erradication of viruses in populations. Amer Jour Ep 1979; 109: 103-123.

13. Glass $G$., Willson $y$ Gottman $S$.: Design and analysis of time series experiment. Colorado Ass Unìversity Press 1974; 237 .

14. Pumarola A.: Epidemiología y profilaxis de las enfermedades infecciosas, en Pumarola A., Torres A., Garcja I. y Piedrola G., 1984. Microbiología y Parasitología médica. Salvat Editores S.A. Barcelona 1984; 308-318. 